\title{
Isotropic Spin Trap EPR Spectra Simulation by Fast Fourier Transform (FFT)
}

\author{
S. LAACHIR ${ }^{1}$, M. MOUSSETAD ${ }^{2}$, R. ADHIRI $^{2}$, and A. FAHLI ${ }^{1}$ \\ ${ }^{1}$ UFR S.I.R.I,Université Hassan II-Mohammedia Faculté des Sciences \\ Ben M'sik, B.P 7955 Casablanca, Morocco \\ ${ }^{2}$ L.P.S.C.M,Université Hassan II-Mohammedia Faculté des Sciences \\ Ben M'sik, B.P 7955 Casablanca, Morocco
}

Received 29 January 2005; Accepted 9 March 2005

\begin{abstract}
The detection and investigation of free radicals forming in living systems became possible due to the introduction of the method of spin traps. In this work, the electron spin resonance (ESR) spectra of DMPO/HO. and MGDFe-NO adducts are reproduced by simulation, based on the Fast Fourier Transform (FFT). The calculated spectral parameters as the hyperfine coupling constants, agree reasonably with the experimental data and the results are discussed.
\end{abstract}

Key words: FFT, Simulation, ESR, spin trap, free radicals

\section{Introduction}

The appearance of nitroxyl radicals became a decisive event for solution of the problem of detection and investigation of free radicals forming in living systems. The detection of radicals became possible due to the introduction of the method of spin traps. The essence of the method consists in the following: a compound that is no nitroxyl radical but has a structure close to nitroxyl radical (spin trap) interacts with a free short-life radical and transforms to a long-life nitroxyl radical (spin adduct) whose EPR spectrum is unique for the given radical or family of radicals. The spin trapping technique involves the addition of the reactive free radical across the double bond of a diamagnetic "spin trap" to form a much more stable free radical, a "radical adduct," which can then be examined with ESR. By their chemical nature, spin traps can be attached to two basic classes: nitrons and nitroso compounds. C-phenyl-N-tret-butyl nitron (PBN), as it follows from its name, is attached to nitrons. One more popular spin trap is 5,5-dimethyl-pirrolin-1-oxyl (DMPO) that is also attached to nitrons ( Fig 1 ). On the other hand, Fast Fourier Transform (FFT), as the name suggests, is a fast and efficient way of computing Discrete Fourier Transform (DFT). This algorithm was independently presented by Cooley-Tukey in $1965^{1}$ which reduces the number of computations from something on the order of $\mathrm{N} 2$ to N $\operatorname{logN}$. There are basically two type of Tukey-Cooley FFT algorithms in use: decimation-in- 
time and decimation-in-frequency. The algorithm is simplified if $\mathrm{N}$ is chosen to be a power of 2 , but it is not a requirement. FFT is a muti grid algorithm to compute the Fourier transform of a function 166

LAACHIR et al.,

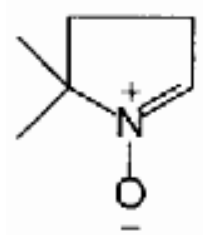

DMPO

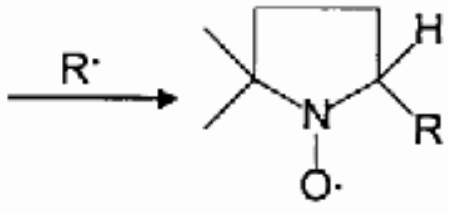

DMPO/R

Figure 1. Spin trapping of the radical R. with DMPO

The technique has many advantages for simulating solution spectra. Among advantages of this method is that complicated spectra can be simulated very quickly and with very little computer memory by performing convolutions in frequency space. There are essentially no limitations on the number of lines which can be simulated. In fact, the efficiency of the algorithm increases as the number of nuclei increases. In this work, a program has been developed for simulation of spin traps and free radicals EPR spectra using the FFT.

\section{Calculation}

FFT is used to generate the EPR spectra. Every simulated spin trap or free radical EPR spectrum data is represented as a complex number with a "real" part and an "imaginary" part. For the simulated free radical EPR spectrum data, the "imaginary" component of the data is zero, and the "real" value represents the strength of the spin trap or the free radical EPR signal, so, only the real parts of the Fourier transforms of the lineshape functions are taken into account The signal position $\mathrm{Hr}$ can be determined using the formula below:

$$
\mathrm{Hr}(\mathrm{G})=\frac{0.714484 \mathrm{v}(\mathrm{MHz})}{\mathrm{g}}
$$

Where is the microwave frequency and $\mathrm{g}$ is the g-value, for free electron, $\mathrm{e}$, has the value close to two: ge $=2.0023193$. First, our simulation program reads an input file (Fig 2). This file included the isotropic g- value, the frequency, the total number of nuclei plus their nuclear spin numbers, the hyperfine splitting parameters, the scan range, the number of data points $(\mathrm{N})$ and finally the linewidth $\mathrm{W}$, which is the first-derivative peak to peak. Next, the program is based on a series of iterative steps that eliminate redundant operations. The iterative step sequentially combines the data into progressively larger weighted sums of data. This iteration may be used to reduce the computation whenever $\mathrm{N}$ is a composite number. The most advantage from the program is gained by selecting $\mathrm{N}$ as a power of a small number. When $\mathrm{N}=2 \mathrm{M}$, where $\mathrm{M}$ is the size of array, input and output arrays all must have a common size. Most of the time, the FFT is used for strictly real-valued inputs.

\section{Results and Discussion}

EPR spin trapping with DMPO

In previous experimental work has been described elsewhere ${ }^{2}$. The formation of DMPO/HO. adducts during the reaction between $\mathrm{H} 2 \mathrm{O} 2$ and Wild-type (WT)-Superoxide dismutase (SOD). ESR spectrum was recorded at room temperature on a Varian E-109 spectrometer operating at $9.5 \mathrm{GHz}$.

In the present paper, we are concerned with simulation, based on Fast Fourier Transform, of ESR spectra. As shown in Fig 3, the spectrum of the DMPO- hydroxyl adduct (DMPO/HO.) consists of four lines with an intensity ratio of 1:2:2:1. Note that, the lineshape signals is usually (first derivative) Lorentzian or Gaussian or some mixture of these. The hyperfine couplings to take into account are 
those relative to the hydrogen $1 \mathrm{H}$ and the nitrogen $14 \mathrm{~N}$, the nuclear spin of the oxygen equals zero. The ESR spectrum is consistent with that of an unpaired electron (of spin oxygen equals zero.

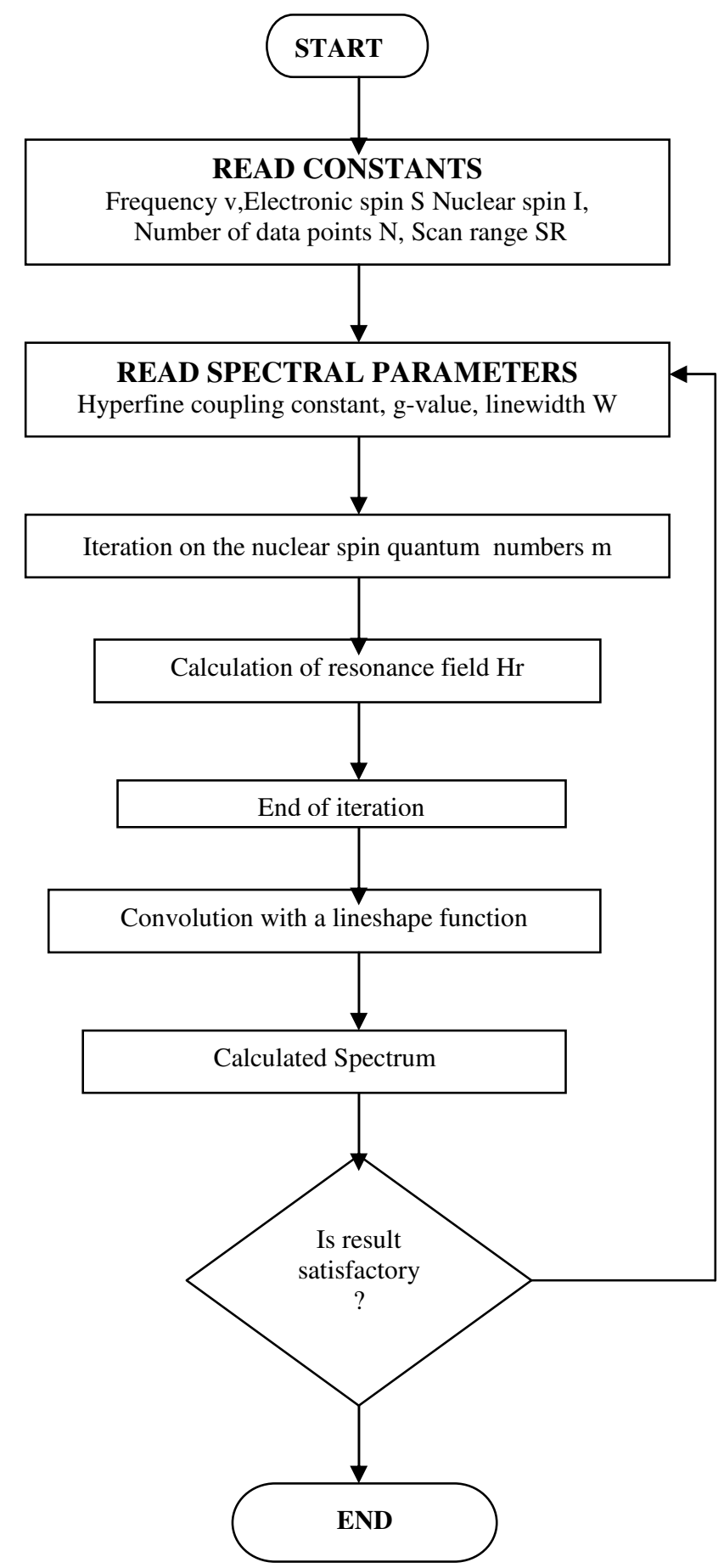


Figure 2. Diagram of the program used for Isotropic Spin Trap EPR Spectra simulation<smiles>CC1(C)CCC(O)N1[O-]</smiles>

\section{DMPO/HO'}<smiles>CCC1CC2(C)CC3(C)CCCC3C2(C)C1C</smiles>

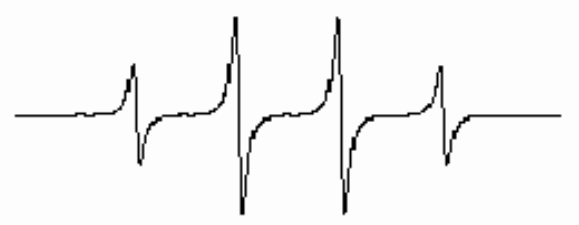

B

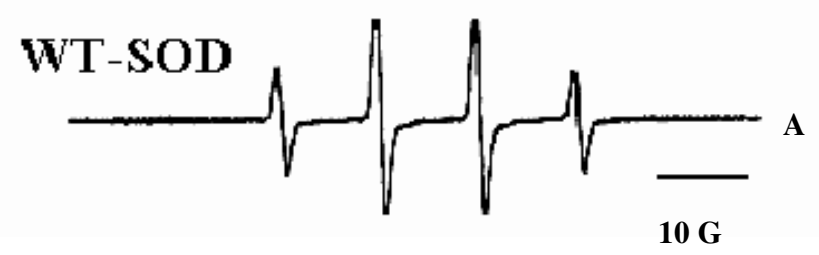

Figure 3. Chemical structure and ESR spectra of DMPO/HO. adduct : (A) Experimental spectrum; (B) Simulated spectrum. The hyperfine coupling constants are provided in the Table 1.

The ESR spectrum is consistent with that of an unpaired electron (of spin 1/2) interacting with one nucleus (nitrogen) of spin 1 and one nuclei (hydrogen) of spin 1/2. ESR spectrum was simulated at Xband with frequency of $9.5 \mathrm{GHz}$, linewidth : $\mathrm{W}=1 \mathrm{G}$, scan range : $\mathrm{SR}=80 \mathrm{G}$, and number of data points : $N=2048$. Both Gaussian and Loretzian lineshapes are supported. The values of the hyperfine constants of nitrogen and hydrogen, respectively, aN and aH are listed in Table 1. These calculated values are in good agreement with the splitting constants of other DMPO-OH adduct by DMPO-4

EPR spectra of $N O$ trapping using $F e-M G D$

Recall that, in a recent work ${ }^{5}$, EPR measurements of Nitric oxide (NO) generation in the NO synthasetransfected human embryonic kidney 293 cells (HEK 293) employed the NO trap Fe-N-methyl-Dglucamine dithiocarbamate (Fe-MGD), stimulation with the Ca2+ ionophore A23187 (1 $\mu \mathrm{M})$ triggered the generation of a prominent triplet spectrum. EPR spectrum was recorded with a microwave frequency of $9.77 \mathrm{GHz}$. On the other hand, the triple peak shape of the spectrum (Fig 4) arises from the interaction of the unpaired electron with the nitrogen nucleus of NO. The three values of nuclear spin quantum number $\mathrm{m}(-1,0$ and 1$)$ can be used to calculate the resonant field positions of the three peaks (Equation 1). For example, the center peak corresponds to $m=0$. Similarly, the right 
and left peaks correspond to $\mathrm{m}=1$ or -1 , respectively. Therefore, the difference, in Gauss, between the center peak and either of the outer peaks is simply: aN ESR spectrum was simulated at X-band

with frequency of $9.77 \mathrm{GHz}$, linewidth : $\mathrm{W}=6.3 \mathrm{G}$, scan range $: \mathrm{SR}=60 \mathrm{G}$, and number of data points $: \mathrm{N}=2048$. Both Gaussian and Loretzian lineshapes are supported. The EPR signal is characterized by an isotropic triplet signal at $\mathrm{g}=2.043$ and $\mathrm{aN}=12.75 \mathrm{G}$. These calculated values are in good agreement with the experimental splitting constants ${ }^{5,6}$ (Table 1).

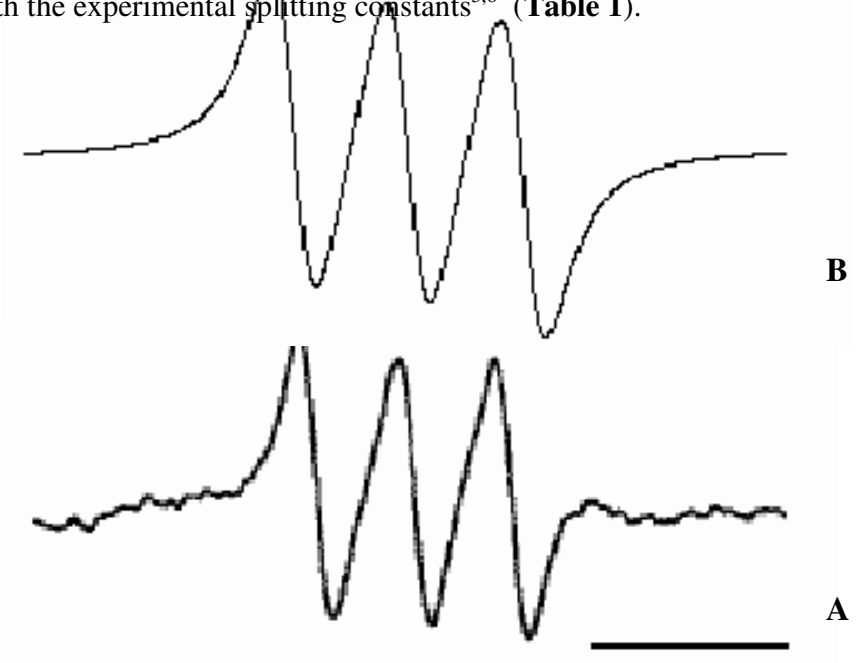

25G

Figure 4. EPR spectra of NO trapping using Fe-MGD: (A) Experimental spectrum; (B) Simulated spectrum . The hyperfine coupling constant is provided in the Table 1.

Table 1. EPR hyperfine coupling constants of DMPO-OH and MGD-Fe-NO adducts

\begin{tabular}{llll}
\hline Spin adduct & AN $(\mathrm{G})$ & Ан $(\mathrm{G})$ & Ref \\
\hline DMPO-OH & 14.9 & 14.9 & $3-4$ \\
& 15 & 15 & 2 \\
& 15.22 & 15.22 & This work \\
\hline \multirow{2}{*}{ MGD-Fe-NO } & 12.7 & -- & $5-6$ \\
& 12.75 & --- & This work \\
\hline
\end{tabular}

Conclusion and Perspectives

In this work, we applied Fast Fourier Transform (FFT) to simulate the ESR spectra of DMPO/HO. and MGD-Fe-NO adducts. We found that the spectra obtained by simulation are in good agreement with the experimental ones. By this program, almost all the free radicals ESR spectra can be simulated The work concerned with a quantum approach based on the spin Hamiltonian is in progress ${ }^{7}$

\section{References}

1 Cooley J W and Tukey J W, An algorithm for the machine calculation of complex Fourier series, Mathematics of Computation, 1965, 19, 297-301.

2 Singh R. J, Karoui H, Gunther M R, Beckman J S, Mason R P and Kalyanaraman B, Proc. Natl. Acad. Sci. USA, 1998 95, 6675-6680 
3 Buettner G R, Free Radical Biology \& Medicine, 1987, 3, 259-303.

4 Arya P, Stephens J C, Griller D, Pou S, Ramos C L, Pou W S and Rosen G M, J. Org. Chem., 1992, 57, 2297-2301.

5 Xia Y, Dawson V L, Dawson T M, Snyder S H and Zweier J L, Nitric oxide synthase generates superoxide and nitric oxide in arginine-depleted cells leading to peroxynitritemediated cellular injury. Proc. Natl. Acad. Sci. USA, 1996, 93, 6770-6774

6 S. Nedeianu and T. Pali. EPR spectroscopy of common nitric oxide - spin trap complexes. Cell. Mol. Biol. Lett. 2002, 7, 142- 143.

$7 \quad$ S. Laachir et al. 


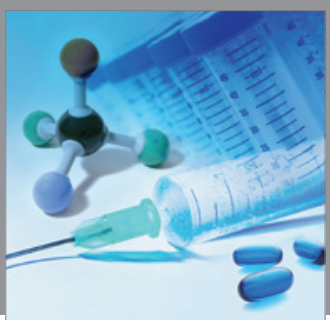

International Journal of

Medicinal Chemistry

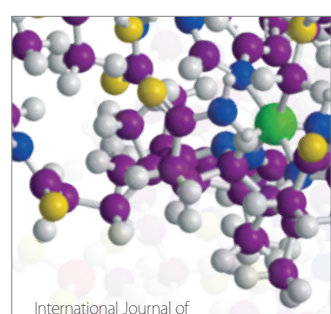

Carbohydrate Chemistry

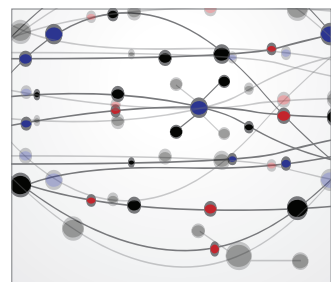

The Scientific World Journal
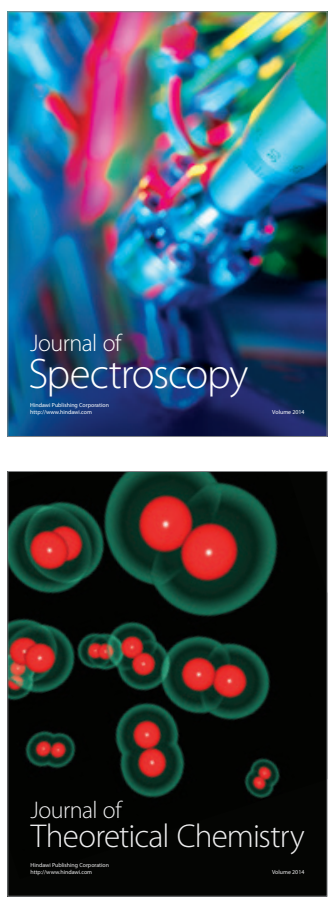
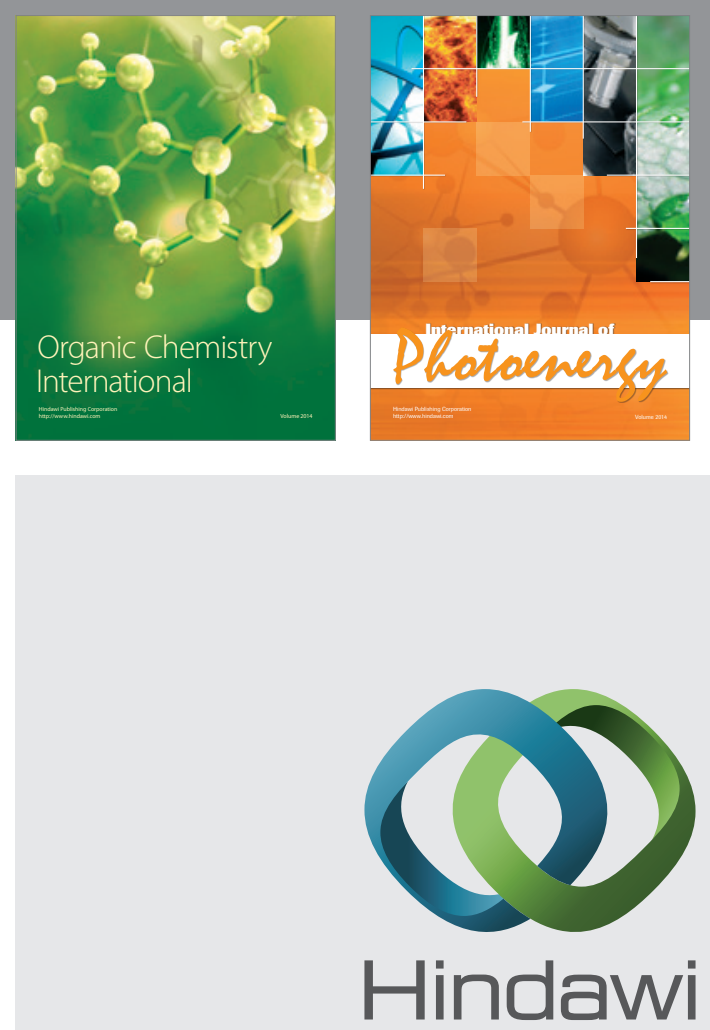

Submit your manuscripts at

http://www.hindawi.com
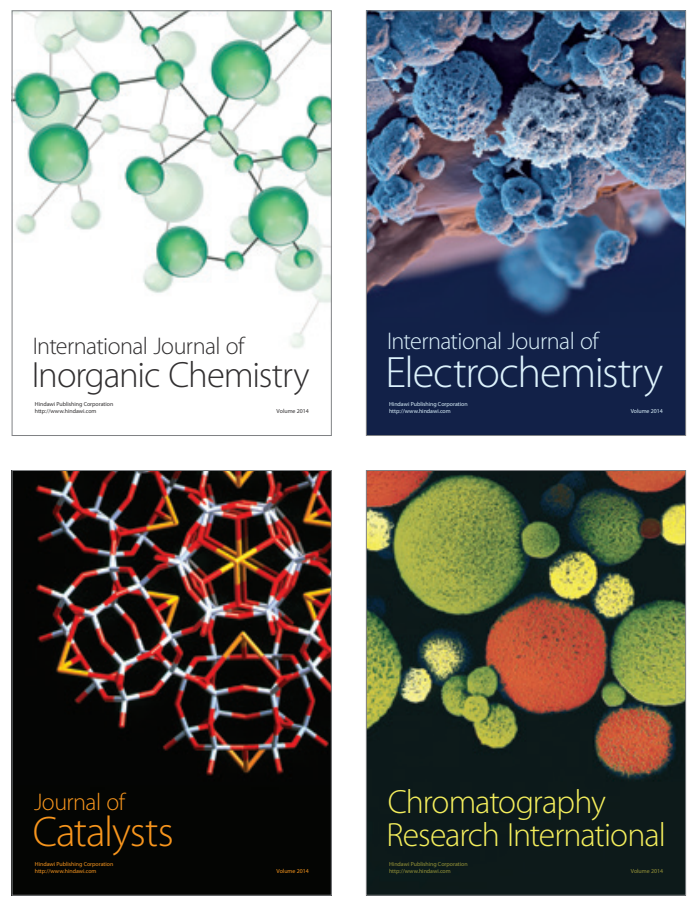
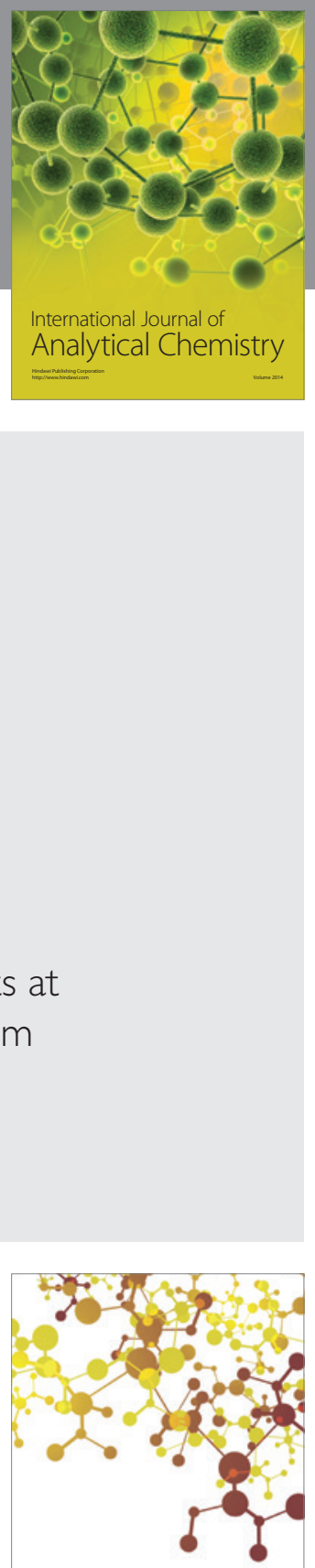

Journal of

Applied Chemistry
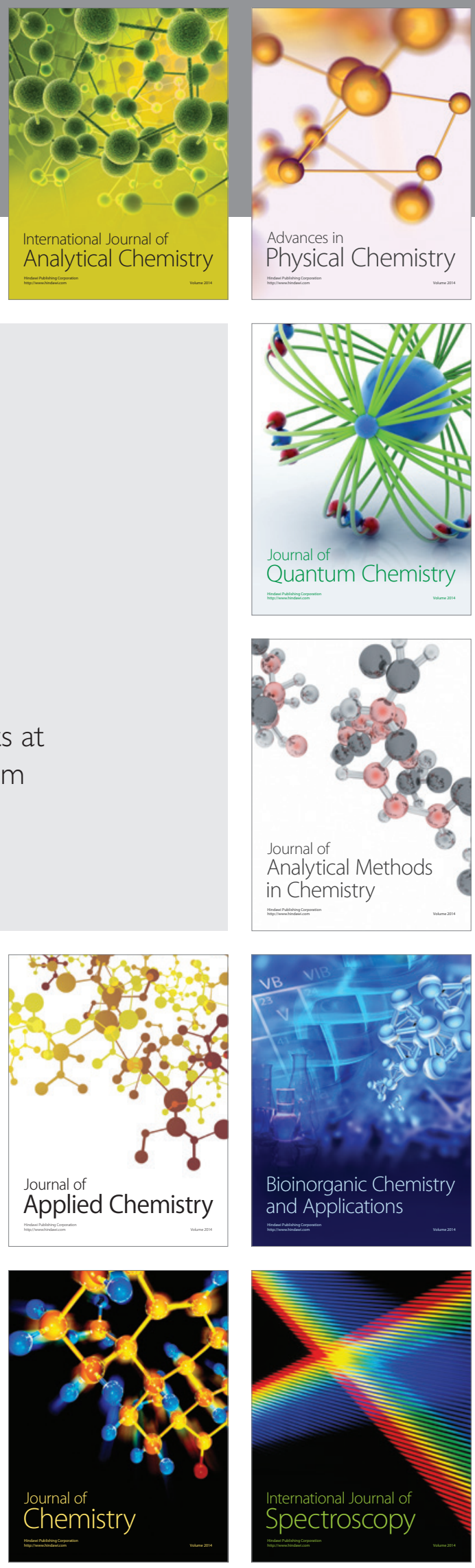